\title{
Real-time genomic profiling of histiocytoses identifies early-kinase domain BRAF alterations while improving treatment outcomes
}

Lynn H. Lee, ${ }^{1}$ Anjelika Gasilina, ${ }^{2}$ Jayeeta Roychoudhury, ${ }^{3}$ Jason Clark, ${ }^{3}$ Francis X. McCormack, ${ }^{4}$ Joseph Pressey, ${ }^{1}$ Michael S. Grimley, ${ }^{3}$ Robert Lorsbach, ${ }^{5}$ Siraj Ali, ${ }^{6}$ Mark Bailey, ${ }^{6}$ Philip Stephens, ${ }^{6}$ Jeffrey S. Ross, ${ }^{6}$ Vincent A. Miller, ${ }^{6}$ Nicolas N. Nassar, ${ }^{2}$ and Ashish R. Kumar ${ }^{3}$

${ }^{1}$ Cancer and Blood Diseases Institute, Division of Oncology, Cincinnati Children's Hospital Medical Center, ${ }^{2}$ Cancer and Blood Diseases Institute, Division of Experimental Hematology and Cancer Biology, Cincinnati Children's Hospital Medical Center, ${ }^{3}$ Division of Bone Marrow Transplantation and Immune Deficiency, Cincinnati Children's Hospital Medical Center, ${ }^{4}$ Department of Internal Medicine, University of Cincinnati College of Medicine, ${ }^{5}$ Division of Pathology, Cincinnati Children's Hospital Medical Center, Cincinnati, Ohio, USA. ${ }^{6}$ Foundation Medicine, Cambridge, Massachusetts, USA.

Many patients with histiocytic disorders such as Langerhans cell histiocytosis (LCH) or ErdheimChester disease (ECD) have treatment-refractory disease or suffer recurrences. Recent findings of gene mutations in histiocytoses have generated options for targeted therapies. We sought to determine the utility of prospective sequencing of select genes to further characterize mutations and identify targeted therapies for patients with histiocytoses. Biopsies of 72 patients with a variety of histiocytoses underwent comprehensive genomic profiling with targeted DNA and RNA sequencing. Fifteen patients ( $21 \%$ ) carried the known BRAF V600E mutation, and 11 patients $(15 \%)$ carried various mutations in MAP2K1, which we confirm induce constitutive activation of extracellular signal-regulated kinase (ERK) and were sensitive to inhibitors of mitogenactivated protein kinase kinase (MEK, the product of MAP2K1). We also identified recurring $A L K$ rearrangements, and 4 LCH patients with an uncommon in-frame deletion in BRAF (N486_P490del or N486_T491>K), resulting in constitutive activation of ERK with resistance to V600E-specific inhibitors. We subsequently describe clinical cases where patients with aggressive multisystem LCH experience dramatic and sustained responses to monotherapy with either dabrafenib or trametinib. These findings support our conclusion that comprehensive genomic profiling should be regularly applied to these disorders at diagnosis, and can positively impact clinical care.

Conflict of interest: S. Ali, M. Bailey, P. Stephens, V.A. Miller, and J.S. Ross are employees of Foundation Medicine Inc.

Submitted: July 11, 2016

Accepted: January 3, 2017

Published: February 9, 2017

Reference information: JCI Insight. 2017;2(3):e89473. https:// doi.org/10.1172/jici.nsight.89473.

\section{Introduction}

The histiocytoses are rare, clinically variable neoplastic disorders characterized by a clonal proliferation of cells of the monocyte-dendritic lineage (1). The most common and well-known is Langerhans cell histiocytosis $(\mathrm{LCH})$ wherein the neoplastic cells resemble epidermal Langerhans cells. Although typically encountered in children, $\mathrm{LCH}$ does occur in adults. In contrast, the related Erdheim-Chester disease (ECD) more typically affects adults $(1,2)$. Clinical manifestations of histiocytoses are highly variable, although certain tissues and organs are more frequently affected such as bones, skin, lungs, and liver (1). While local control and combination chemotherapy can be curative in some, many patients experience disease recurrence, and others develop irreversible end-organ injury associated with significant morbidity such as diabetes insipidus (DI) and anterior pituitary dysfunction, neurodegeneration, and end-stage hepatic cirrhosis (3-6). The etiopathogenesis of LCH and the other histiocytoses remained unclear for decades, until the identification of recurring BRAF V600E mutations (7, 8 ) and more recently mutations in the downstream gene $M A P 2 K 1$ in LCH and ECD $(9,10)$. Several anecdotal reports and case series describe the successful treatment of patients with LCH or ECD with BRAF V600E-specific inhibitors, suggesting that targeted inhibition is a valid therapeutic strategy in the histiocytic neoplasms (11-14). 
Given the high risk of recurrence and the inherent unpredictable response to therapy in some patients, we sought to characterize the mutations in the histiocytic lesions of patients in real time, with the goal of identifying alternative treatments for patients with inadequate response to standard therapies. As a secondary goal, we aimed to further characterize the biological effects of $M A P 2 K 1$ mutations found in $\mathrm{LCH}$ and $\mathrm{ECD}$, and determine their susceptibility to targeted inhibition.

\section{Results}

Genomic profiling identifies alterations in majority of patients with histiocytoses

Seventy-two patient samples underwent targeted DNA and RNA sequencing as described. Diagnoses included LCH, ECD, juvenile xanthogranuloma (JXG), Rosai-Dorfman disease (RDD), and histiocytosis not otherwise specified (NOS) (Supplemental Tables 1 and 2; supplemental material available online with this article; https://doi.org/10.1172/jci.insight.89473DS1). Figure 1 graphically represents the distribution and patterns of genomic changes identified. Fifteen patient samples (21\%) harbored the BRAF V600E point mutation. Four patients carried a potentially novel 5 or 6 amino acid in-frame deletion (indel) in BRAF (N486_P490del in 3 patients and N486_T491>K in 1) and these patients all carried a diagnosis of LCH. Eleven patient samples (15\%) harbored activating indels or point mutations in MAP2K1. Six patient samples harbored mutations in $K R A S$, with 1 sample containing 2 point mutations, and another containing a point mutation as well as a $K R A S$ amplification. The mutations in $B R A F$ and $M A P 2 K 1$ were always mutually exclusive. Two patients harbored missense mutations in both $N R A S$ and $B R A F$, albeit with disparate allele frequencies $(8 \%$ and $1 \%$, respectively, for patient sample A-ECD- 47 , and $13 \%$ and $21 \%$, respectively, for patient A-LCH-71). The sample from patient A-LCH-71 also demonstrated the presence of a KRAS mutation with a variant allele frequency (VAF) of only 1\%. Two adult patient samples (1 ECD and 1 histiocytosis NOS) contained amplification of $M A P 3 K 1$ and $P D G F R A$, respectively. In 8 patients, mutations in TET2, DNMT3A, or ASXL1 were found, alone or in combination with mutations in $B R A F$.

RNA sequencing also identified several patients with recurrent $A L K$ gene fusions, previously described in other malignancies and recently also identified in ECD (11). Specifically, 3 patients carried the KIF5B$A L K$ rearrangement: 2 (1 pediatric and 1 adult) with histiocytosis NOS and 1 pediatric patient with JXG. Additionally, lesional biopsy of an adolescent patient with histiocytosis NOS displayed the EML4-ALK rearrangement. Other kinase fusions detected in adult patients with histiocytosis NOS lesions include TPR-NTRK1, and 1 patient with a ZNF697-NOTCH2 fusion. Two patients in this data set have been previously reported in other publications: the histiocytosis NOS patient with the BRAF-CLIP2 fusion was also described in the study by Diamond et al. (11) and one NOS patient bearing an ETV3-NCOA2 fusion was referenced by Brown et al. (15). Our data set, however, includes another adult patient with the diagnosis of $\mathrm{LCH}$ who also exhibited the ETV3-NCOA2 fusion, suggesting that this is a recurring gene rearrangement and is not restricted to indeterminate histiocytosis. The remaining patients in our data set are to the best of our knowledge unique and have not been published elsewhere.

We thus classify the mutations identified in this study into different functional groups. The majority of patients had mutations that presumably directly activate the RAS/RAF/MEK/ERK pathway, namely mutations in $B R A F, M A P 2 K 1$, but also point mutations in $K R A S, 1 B R A F$ fusion, $A L K$ rearrangements, and amplifications of PDGFRA, KIT, and MAP3K1. The ZNF697-NOTCH2 rearrangement does not appear to be functional, as the fusion joins the 5'-untranslated region of ZNF697 out of frame with exon 26 of NOTCH2 (Supplemental Figure 1). The sample expressing this fusion also carried PDGFRA and KIT amplifications, which we speculate are more likely to be driver mutations. Other recurring genomic alterations included mutations involving p53 or its regulatory proteins, and loss of $C D K N 2 A / B$. Of the 72 patients included in this cohort, $16(22 \%)$ did not have what we considered a candidate driver mutation, namely a gene fusion or alteration affecting the MAP kinase pathway.

\section{Knowledge of genomic alterations improves clinical outcomes}

We describe 1 adult and 3 pediatric patients where genomic characterization had a direct positive impact on clinical management.

Case 1. A 22-month-old female was transferred to our hospital for management of refractory LCH complicated by secondary hemophagocytic lymphohistiocytosis (HLH). She initially presented at 4 months of age with a rash confirmed by biopsy to be cutaneous LCH. Her parents initially declined treatment owing 


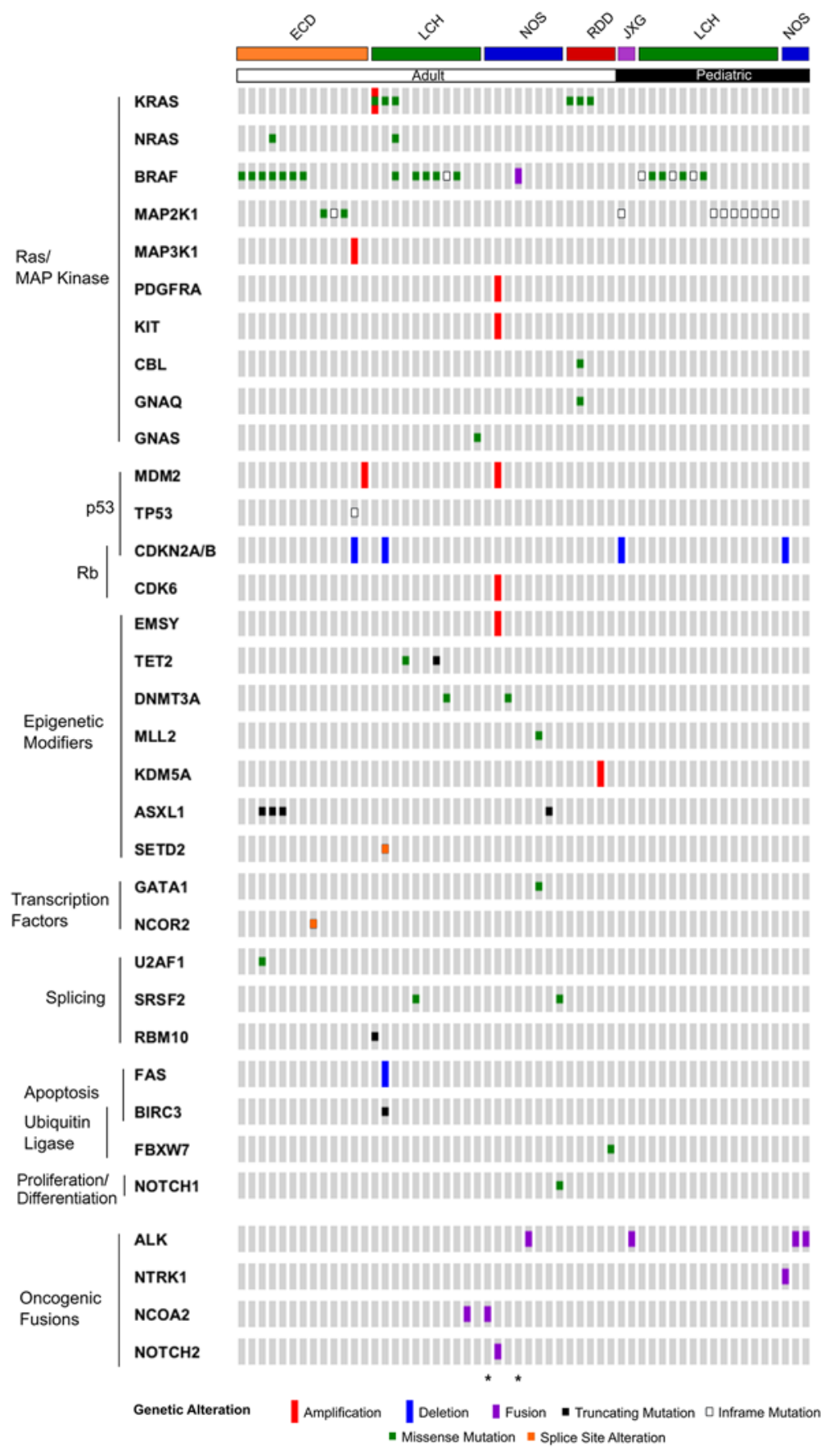

Figure 1. Comutation plot demonstrating the variety of likely pathogenic mutations and gene fusions detected through targeted sequencing. Single columns represent individual patients with the diagnosis identified in the first row. Samples are grouped by disease type and age group as indicated in horizontal bars. Asterisks at the bottom represent 2 previously reported patients as described in the text. Basic plot created using cBioPortal $(39,40)$. to modest spontaneous improvement in her cutaneous disease. However, her skin lesions progressed and she eventually developed persistent fever and marked pancytopenia at 4 months after diagnosis. Her soluble IL-2 receptor was also substantially elevated $(14,914$ units $/ \mathrm{ml}$; normal range 334-3,026). Imaging revealed an expansile bony lesion involving the right basal skull which was metabolically active on fluorodeoxyglucose positron emission tomography (FDG-PET) scan, and bone marrow evaluation revealed frequent hemophagocytic forms with phagocytosed red blood cells, myeloid progenitors, and platelets (Figure 2, A and B). Notably, the bone marrow was hypercellular and effaced with CD163-positive cells (Figure 2, C and D). These cells were negative for CD1a though, and showed nonspecific S100 staining, suggesting lack of direct marrow infiltration by Langerhans cells. Treatment was initiated with vinblastine and prednisone. However, fever and pancytopenia persisted in spite of several weeks of therapy. She was then treated with HLH-directed therapy consisting of dexamethasone, etoposide, and cyclosporine, but in vain. Upon transfer to our hospital, we attempted retrieval with clofarabine, based on the presumption that her HLH was being driven by an exaggerated immune/inflammatory response to the underlying $\mathrm{LCH}$. However, her clinical status remained unchanged with persistent fevers and transfusion-dependent cytopenias. A bone marrow evaluation 3 weeks after clofarabine administration demonstrated persistent, extensive hemophagocytosis.

As part of the genomic characterization of her $\mathrm{LCH}$, immunohistochemistry for the BRAF V600E protein was performed on a skin biopsy sample and on the bone marrow as well, using a well-described and validated BRAF V600E-specific antibody (16-18). Within the bone marrow, the majority of the CD1a-negative macrophages were BRAF V600E positive by immunostain (Figure 2, E and F). The mutation was confirmed by comprehensive genomic profiling using the platform described above. Based on this finding and her refractoriness to conventional cytotoxic chemotherapy, we treated her with the BRAF inhibitor dabrafenib $(5 \mathrm{mg} / \mathrm{kg} /$ day divided into 2 doses). Within days of starting dabrafenib, she became afebrile and her skin rash resolved. Shortly thereafter, her peripheral blood counts and inflammatory markers normalized (Figure $2 \mathrm{G}$ ). Her FDG-avid skull lesions improved markedly on imaging after 6 weeks of therapy (Figure 2, $\mathrm{H}$ and I). She is asymptomatic and thriving on dabrafenib with her disease in remission.

Case 2. A 36-year-old previously healthy woman complained of polyuria and polydipsia and was eventually diagnosed with DI. Brain MRI revealed loss of the pituitary-stalk signal. However, since she was otherwise asymptomatic, no treatment was initiated. Over the next few years, she developed short-term memory loss and seizures. She also developed large tumors in her neck and groin, which were FDG-PET avid (Figure 3A). A biopsy confirmed these as LCH. Systemic chemotherapy was recommended; however, she declined treatment owing to concerns regarding its impact on her quality of life. Targeted sequencing 


\section{A}
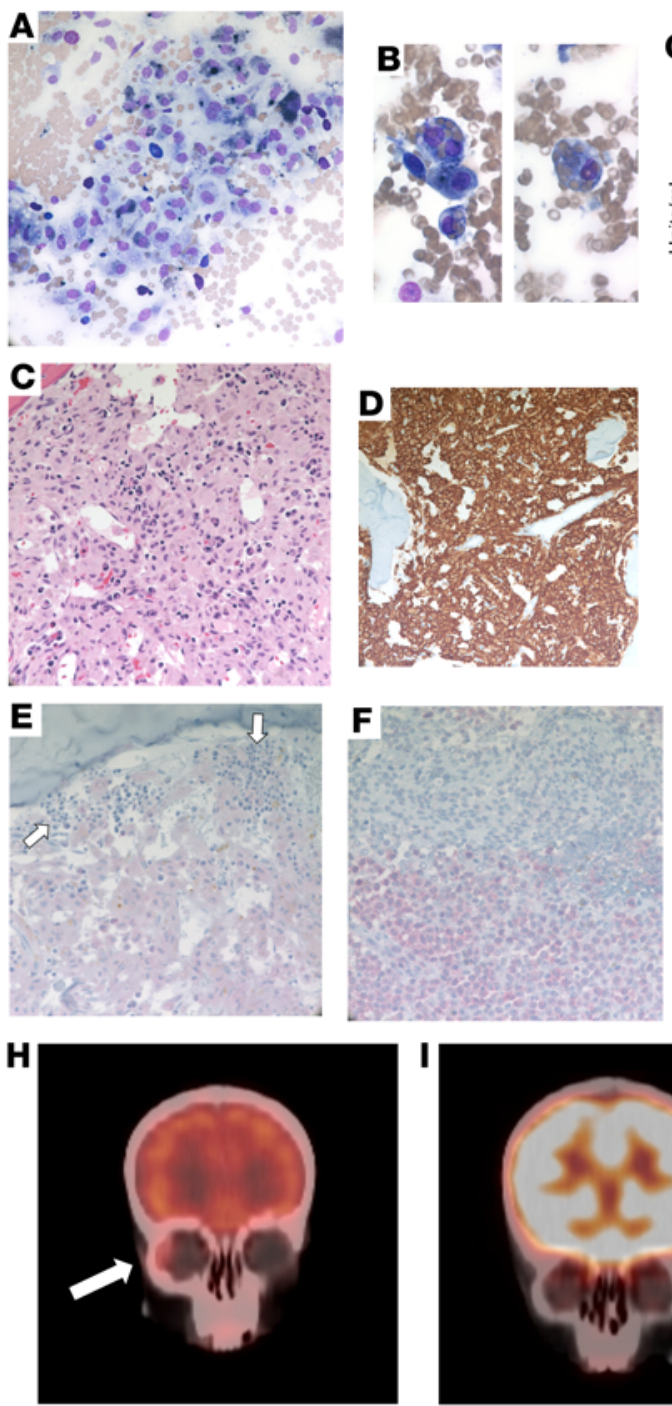

G

Soluble IL2 Receptor
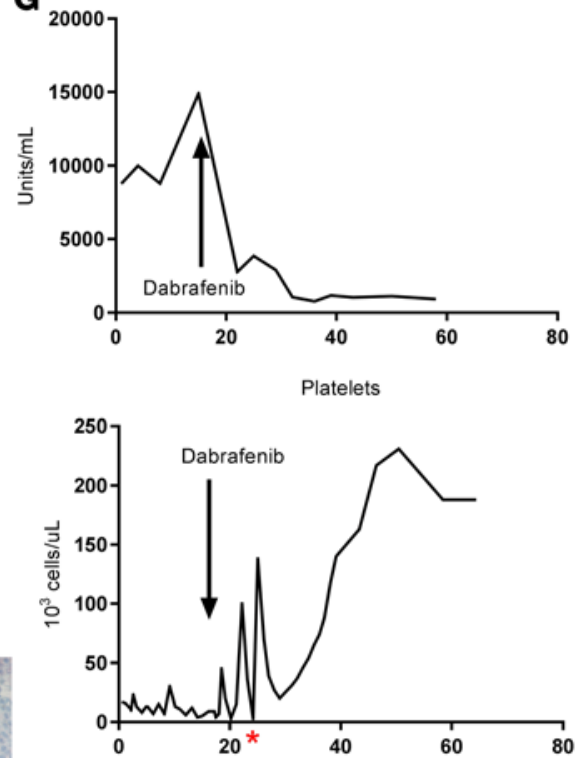

Hemoglobin
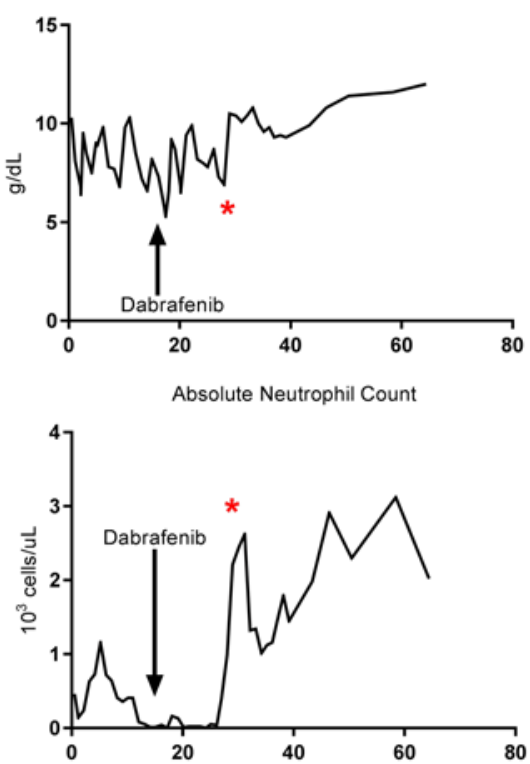

Day of admission

Figure 2. Mutant BRAF is detected in non-Langerhans bone marrow histiocytes and responds to targeted therapy. Patient 1: (A) Most of the cellularity in the bone marrow aspirate was comprised of mature-appearing histiocytes, many of which contain hemosiderin. Few hematopoietic cells were present. (B) Frequent hemo-

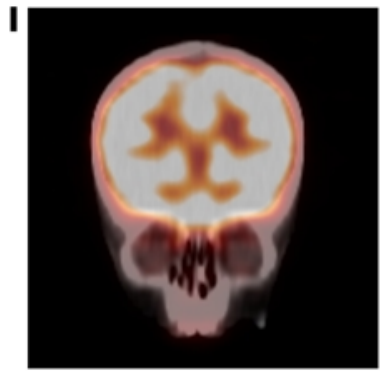
phagocytic cells were present in the aspirate. (C) Histiocytes filled much of the bone marrow in the biopsy. (D) The bone marrow was diffusely positive for CD163 and negative for CD1a and S100 (not shown). (E) The histiocytes were positive for BRAF V600E using a mutation-specific antibody (pink), whereas other hematopoietic cells in the marrow were BRAF negative (arrows). (F) Positive control BRAF immunohistochemistry on a Langerhans cell histiocytosis skin biopsy containing BRAF V600E; note specific cytoplasmic staining (pink) of the neoplastic histiocytes in the lower one-half of the field. (C) Blood counts and soluble IL-2 receptor levels before and after initiation of dabrafenib by day of admission ( $x$ axis). Red asterisks represent the patient's last required transfusion (blood or platelets) or dose of granulocyte colony-stimulating factor, as in the case of absolute neutrophil count. (H) Coronal view of fluorodeoxyglucose-avid basilar skull lesion with orbital involvement (arrow) is markedly improved on followup PET/CT obtained 2 months later (I) after 6 weeks on dabrafenib. Original magnification, $\times 400$ (A and F), $\times 1,000$ (B), $\times 200$ (C-E).

of a biopsy from the neck tumor revealed a $B R A F$ indel (N486_P490del) with a VAF of 14\%. Interestingly, we also found the previously described DNMT3A Y 533C mutation, although with a much lower VAF (1\%). Given that this $B R A F$ indel had been detected in 3 other patients with $\mathrm{LCH}$, we suspected this mutation to be causative. This deletion had been previously reported in the ovarian cancer cell line OV90 (19) and the Me1537 melanoma cell line as well (20). Experimental evidence indicated that akin to BRAF V600E, this mutation also results in constitutive activation of downstream signaling but is insensitive to V600Especific inhibitors (21). We thus elected to treat her with the MEK inhibitor trametinib. Strikingly, within 5 days of initiating treatment, her neck and groin tumors disappeared. Prior to initiation of trametinib, she had partial seizures several times per day. Two weeks after starting treatment, she became seizure free and has remained so for several weeks. Although she still suffers from short-term memory loss, her mood and quality of life have significantly improved. Follow up PET-CT showed her lesions had completely resolved (Figure 3B). She remains in remission at the time of this writing.

Case 3. A 10-month-old child was diagnosed with extensive multisystem LCH involving the skin, soft palate, and spleen, which was characterized by pancytopenia and persistent fevers suspicious for secondary HLH. Similar to the first patient described above, bone marrow evaluation demonstrated marked histiocytic hyperplasia with hemophagocytosis. The histiocytes were CD1a- and S100-negative. Notably, his disease was found to carry the BRAF V600E mutation and the infiltrating histiocytes were BRAF V600E 
A

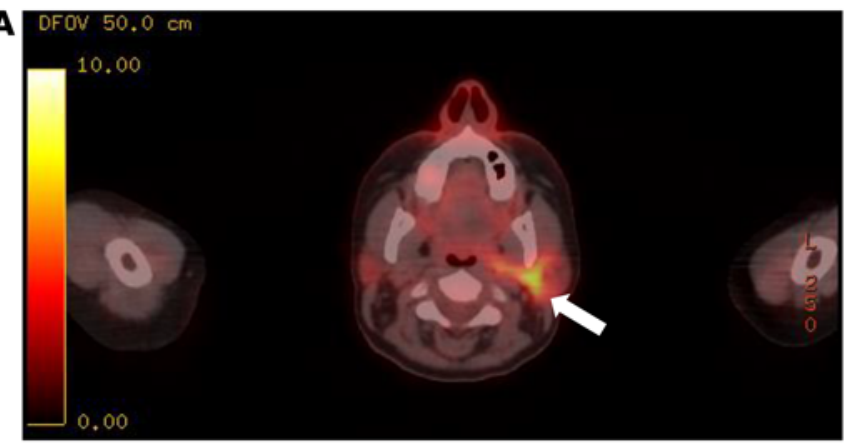

C

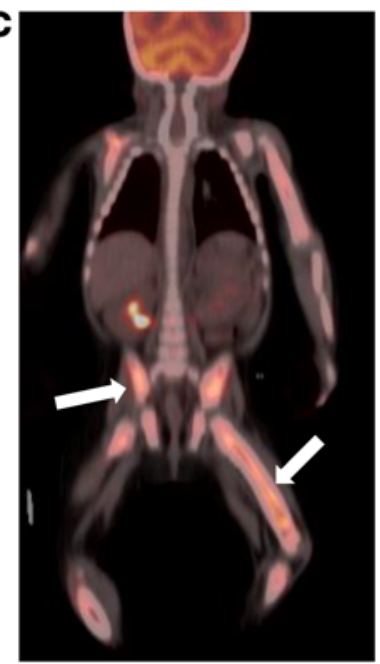

D

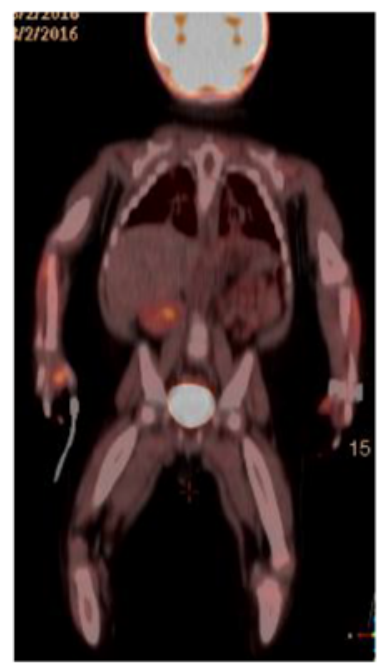

B

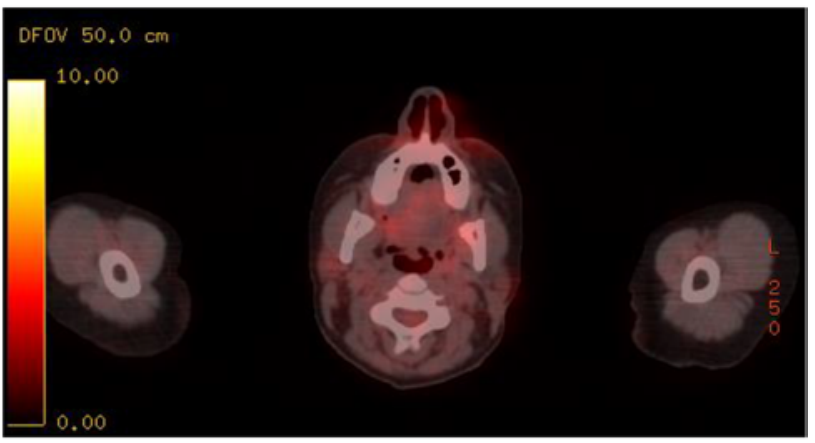

Figure 3. Responses to targeted therapy in Langerhans cell histiocytosis by functional imaging. (A) Patient 2: PET-CT at diagnosis (left) demonstrated markedly fluorodeoxyglucose-avid (FDG-avid) lesions in the left neck and groin (latter not pictured). (B) Follow-up PET-CT after 4 months of therapy demonstrates near-total resolution at sites of disease in the neck. (C) Patient 3: PET-CT obtained upon transfer to our institution demonstrated marked FDC avidity in the skeletal medullary spaces (white arrows) secondary to disease activity, which demonstrated near-total resolution on follow-up imaging (D) 3 months after the start of therapy.

positive on immunostain. Chemotherapy was commenced with vinblastine and prednisone; while his skin and soft palate disease responded, his hepatosplenomegaly and transfusion dependence persisted. These symptoms failed to improve with attempts at salvage chemotherapy with multiple courses of clofarabine, and even the combination of clofarabine and cytarabine. Additionally, splenectomy was performed in an attempt to relieve his transfusion requirement. He was subsequently transferred to our institution for further management. A PET/CT obtained shortly after transfer demonstrated extensive diffuse skeletal FDG uptake (Figure 3C). He also had daily fevers and required red blood cell and platelet transfusions several times per week. We elected to treat him with dabrafenib as well. Similar to the previous patient, his fevers resolved within days of starting dabrafenib, and his red blood cell requirement gradually abated. A followup PET/CT obtained 12 weeks after starting dabrafenib demonstrated near-complete resolution of abnormal FDG uptake initially noted upon transfer (Figure 3D). He continues to occasionally require platelet transfusions, albeit at a much reduced frequency.

Case 4. A 9-month-old male infant was diagnosed with multisystem $\mathrm{LCH}$ with skin and bony disease, as well as liver involvement based on the presence of elevated serum aminotransferases and hepatic FDG avidity on PET. Treatment was initiated with vinblastine and prednisone. While his skin and bone disease responded, he was noted to have a gradual and sustained increase in his alanine aminotransferase (ALT) and alkaline phosphatase as well as increased liver metabolism on FDG-PET scan, consistent with treatment-refractory disease. Therapy was switched to cytarabine but his liver abnormalities persisted and his skin rash and bone lesions reappeared. Targeted genetic profiling of a biopsy sample revealed the presence of a $B R A F$ V600E mutation. Liver biopsy showed loss of normal liver architecture but was negative for CD1a- and S100-positive cells. Salvage therapy with clofarabine was commenced, and while all other visible disease responded, his liver disease persisted with worsening hepatomegaly. In an attempt to determine if the liver disease was directly caused by $\mathrm{LCH}$, immunohistochemistry for the BRAF V600E protein was performed on his liver. Surprisingly, we observed infiltrating macrophagelike mononuclear cells, which were BRAF V600E positive but were CD163 negative (Figure 4, A and B). Based on the persistent liver disease and recurrence of bone disease in the setting of a BRAF V600E 


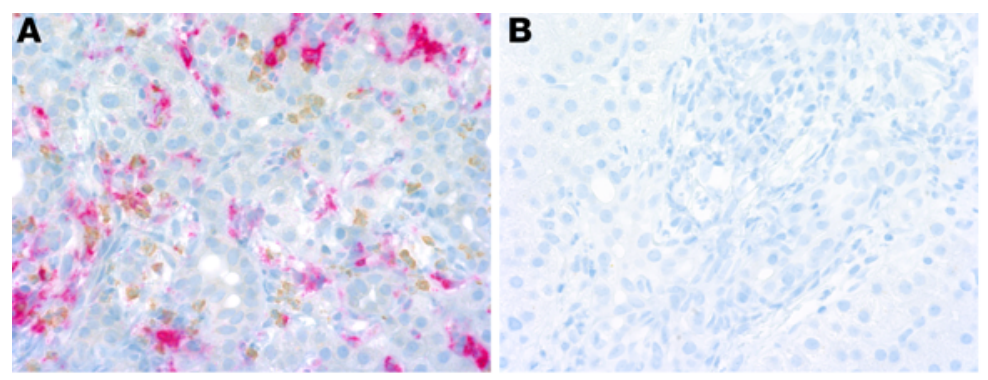

Figure 4. Identification of cells expressing mutant BRAF protein in hepatic involvement by Langerhans cell histiocytosis. (A) Patient 4: Immunohistochemistry for BRAF V600E (brown) and CD163 (red) in representative liver biopsy section demonstrates presence of BRAF V600E mutant protein, but not in cells of macrophage/monocyte lineage (original magnification, $\times 400$ ). (B) Immunostain for CD1a is negative.

mutation, we treated him with dabrafenib. Within a few days his abdominal girth notably reduced. A follow-up PET scan showed resolution of FDG uptake in the liver and all other sites of disease. He is asymptomatic and thriving on dabrafenib.

\section{Mutations in MAP2K1 transform 3T3 cells and result in constitutively active MEK that is sensitive to available inhibitors}

To determine the functional impact of the mutations in $M A P 2 K 1$, we studied NIH/3T3 cells transduced with select MEK mutants stably expressed at equal levels (Figure 5A) because of the lack of a representative in vitro model or cell line. When compared with wild-type MEK, all mutations exhibited increased basal ERK1/2 phosphorylation even without EGF stimulation (Figure 5B). Additionally, the kinetics of ERK phosphorylation appear to be altered in the constitutively active mutants. In cells expressing wild-type MEK, phospho-ERK levels gradually fade despite sustained EGF stimulation, likely as a result of endogenous regulatory phosphatases. The MEK mutants on the other hand result in sustained phosphorylation of ERK, demonstrating the uninterrupted activation of downstream signaling (Figure 5C). As demonstrated by focus formation in culture, the MEK mutations induced growth and features of transformation in 3T3 cells, when compared with wild-type controls (Figure 5, D and E). As a positive control, we compared these results with growth caused by expression of a synthetic mutation, hereby referred to as MEK-DD. This constitutively active version of MEK was derived by substituting residues typically phosphorylated by the RAF kinases (negatively charged serines 218 and 222) with aspartates $(22,23)$. This mutation exhibits transformative properties in vitro $(22,23)$ and the growth effects caused by the MEK mutants described above were comparable to the effects of MEK-DD. Collectively, these results support an oncogenic role for the activating mutations we found in $M A P 2 K 1$.

We next sought to determine the susceptibility of the mutant MEK proteins to inhibition by available inhibitors trametinib, selumetinib (AZD-6244), and PD-0325901. In fibroblasts transformed with the various mutations, we quantified relative levels of phosphorylated ERK1/2 upon treatment with inhibitor or vehicle. We found that ERK1/2 phosphorylation decreased in a dose-dependent manner upon exposure to all MEK inhibitors tested, with efficacy seen even in the nanomolar range. The BRAF V600E inhibitor vemurafenib was used as a negative control, and did not significantly affect ERK1/2 phosphorylation as expected (Figure 6A). Additionally, stably transduced BaF/3 (murine pro-B) cells expressing various MEK mutations showed similar inhibition (Figure 6, B and C). Overall, these data demonstrate that MEK inhibitors effectively decreased ERK1/2 phosphorylation in transformed hematopoietic cells.

\section{Discussion}

Our results from clinical genomic profiling further demonstrate the diversity of genomic lesions that drive $\mathrm{LCH}$ and other histiocytoses. Akin to previous publications describing retrospective whole-exome sequencing $(7,9,10,24)$, we found mutations in $M A P 2 K 1$ and the $B R A F$ V600E point mutation. Additionally, we identified a recurrent novel deletion in $B R A F$, recently characterized biochemically (Supplemental Figure 2) $(21,25)$. This deletion activates downstream ERK, similarly to BRAF V600E, but is resistant to BRAF V600E inhibitors (21). The fact that this mutation was not identified by the previous reports may simply reflect the rarity and heterogeneity of $\mathrm{LCH}$ or a selection bias. Importantly, we were able to incorporate these results directly into patient care, improving the outcomes of patients with multisystem or refractory LCH. In particular, results from genomic profiling helped us treat the patient with the novel BRAF indel, and the infants with multisystem disease and secondary HLH who had failed all previous cytotoxic chemotherapy. All of these patients experienced significant clinical responses with single-agent kinase inhibitors. 
A
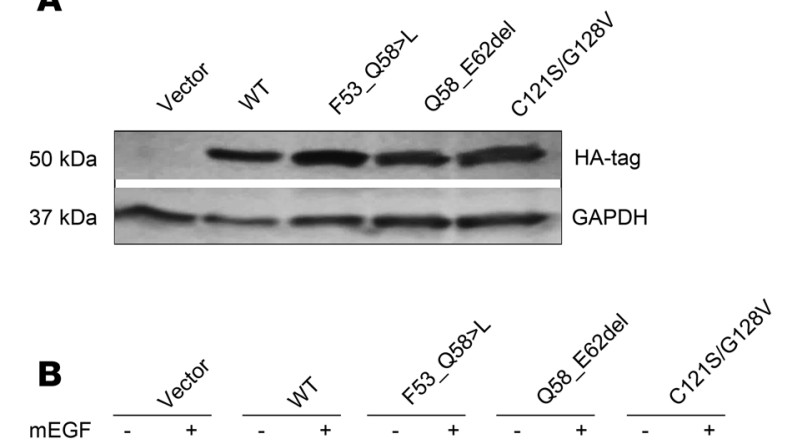

$400 x$

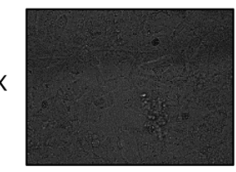

D

$40 x$

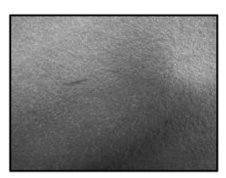

.
MEK-DD

(S218D/S221D)

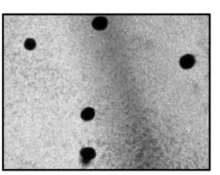

C121S/G128V

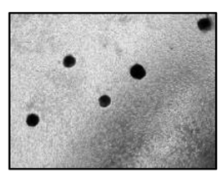

Q58-E62del
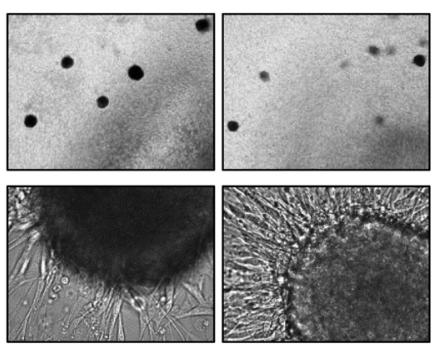

E

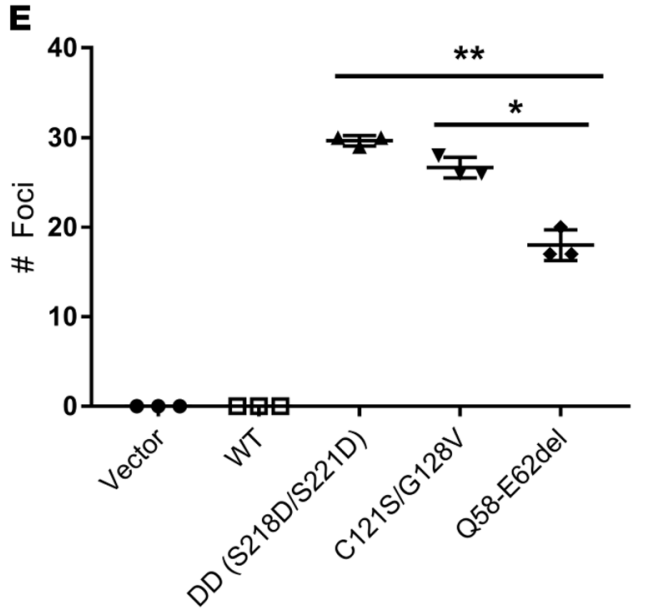

Figure 5. MEK (MAP2K1) mutants demonstrate altered activation kinetics and exhibit signs of transformation in vitro. (A) NIH/3T3 cells were transduced with retroviruses carrying empty vector or HA-tagged MEK constructs. Shown is a representative immunoblot using antibodies against the HA-tag or CAPDH (control) with the various mutations labeled on the top of each lane. WT, wild type. (B) Transduced cells were treated with murine epidermal growth factor (mEGF) and lysates were analyzed by immunoblotting. Representative blots depict phospho-ERK (pERK) and total ERK (tERK) levels in the various 3 T3 clones labeled on the top. Antibodies are labeled to the right with GAPDH used as internal control. (C) 3T3 cells expressing WT and mutant MEK constructs were stimulated with EGF for the indicated time periods; mutant enzymes exhibit prolonged pERK activation in response to sustained EGF stimulation compared with WT MEK. (D) Fibroblasts were plated in monolayer in conventional liquid culture and foci were counted after 21 days. Representative micrographs of cultures expressing the various MEK mutants are shown, labeled on the top, shown at $\times 40$ (upper row) and $\times 400$ (lower row) original magnification. (E) Bar graph depicting the number of foci observed in each condition. Middle horizontal bars represent mean number of foci; error bars represent standard deviation of 3 separate experiments. No foci were seen with WT MEK or vector-only transduced cells. Statistical analysis performed using 1-way ANOVA with Tukey's test post-hoc. ${ }^{*} P<0.001,{ }^{* *} P<0.0001$.

We also identified kinase fusions activating the RAS pathway akin to the findings of Diamond et al. (11). We further investigated the behavior of MAP2K1 mutations in vitro, and showed that these mutations result in activation of the MAP kinase pathway, and are capable of transforming hematopoietic cells. Finally, we demonstrate that mutant MEK is easily inhibited by currently available drugs.

While the current class of MEK inhibitors stabilize the wild-type kinase in its inactive conformation (26), it remains unclear if and how these drugs are effective against mutated forms of MEK. The majority of the mutations we describe occur outside of the kinase domain, activating MEK by destabilizing a negative regulatory region (Supplemental Figure 3). Despite this, the allosteric inhibitors still appear to suppress the activity of mutated forms of MEK, decreasing levels of phosphorylated ERK1/2. Interestingly, in our studies the compound mutant MEK C121S/G128V, previously described by Brown et al., retains susceptibility to trametinib (9). Nelson et al. characterized the similar C121S/G128D mutations in cis, and showed that the decrease in ERK phosphorylation with trametinib was less than that seen in single amino acid mutants at residues 121 and 128 (28). However our results show that the compound mutant MEK C121S/G128V still retains relative susceptibility to MEK inhibition which is comparable to the effect seen with other mutants characterized in our study. These previous studies either looked at cell growth in BRAF V600E-mutant cell lines augmented with MAP2K1 mutations (27) or characterized ERK phosphorylation in cell-free kinase assays (28). We studied MAP kinase signaling in a cellular context, without the potential signaling influence from activated $B R A F$, which might explain this discrepancy. These results suggest that the current class of MEK inhibitors may be 
A

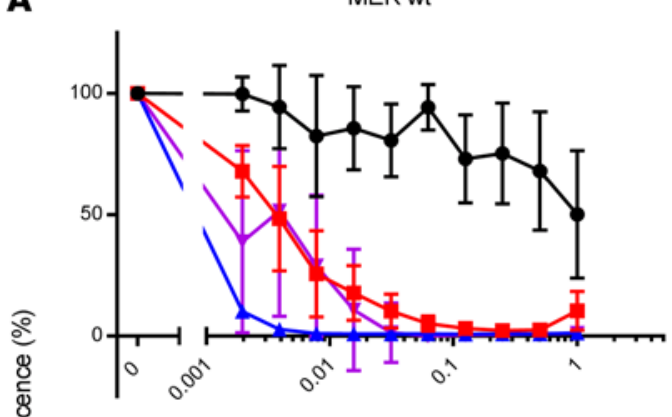

MEK Q58_E62del

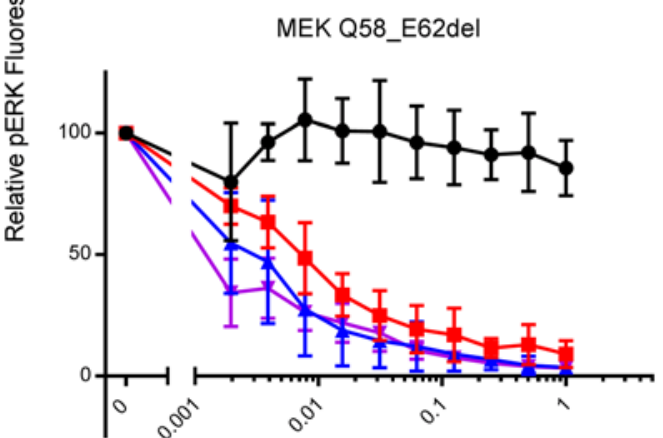

MEK F53_Q58>L

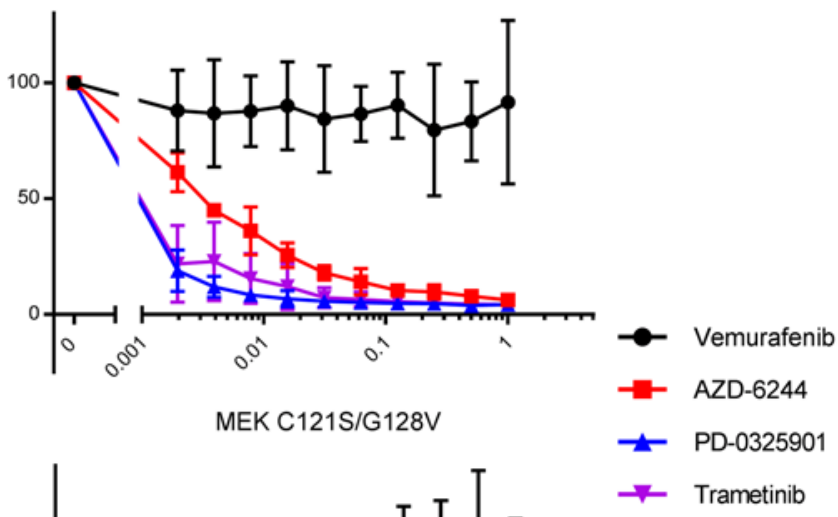

Drug concentration $(\mu \mathrm{M})$

B

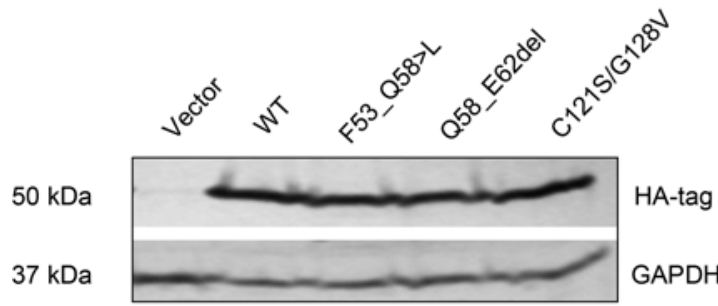

C
pERK $1 / 2$ tERK 1/2

F53_Q58>L

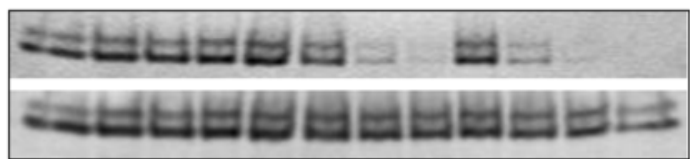

pERK 1/2

tERK $1 / 2$

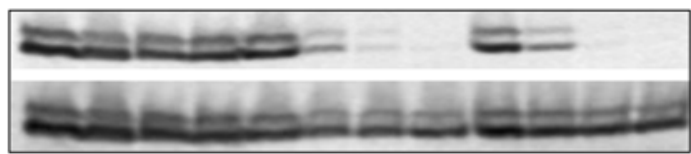

PERK 1/2

tERK $1 / 2$

pERK 1/2

tERK 1/2

Figure 6. Activating mutations in MEK are sensitive to allosteric inhibition. (A) Dose-response curves quantifying phospho-ERK (pERK) activation in transduced NIH/3T3 cells in response to drug treatment. Following drug treatment, cells were fixed and probed simultaneously with fluorescent $p E R K$ and ERK antibodies, and imaged with an Odyssey scanner (LiCor Biosciences). Relative pERK fluorescence ( $y$ axis) was calculated and reflects the percentage change relative to DMSO-treated cells; all values are normalized to total ERK (tERK) expression. Data represent mean of 3 separate experiments; error bars represent standard deviation. (B) Western blot demonstrates stable transduction of BaF/3 cells with pBABE-puro-based empty vector, or WT/mutant HA-tagged MEK constructs. (C) Immunoblots for pERK after 30 minutes of incubation with the indicated drug and concentration show a similar dose-dependent response.

effective for treating patients with $M A P 2 K 1$-mutant histiocytoses. Individual reports of robust and sustained responses to single-agent trametinib in MAP2K1-mutant ECD patients further support this hypothesis (11).

Beyond mutations in the MAP kinase pathway, the role of the co-occurring mutations discovered through our efforts remains unclear. Many of these identified mutations occur in adult patients, and involve genes implicated in the recently described phenomenon clonal hematopoiesis of indeterminate potential (CHIP) such as DNMT3A, TET2, and ASXL1 $(29,30)$. One patient carried both the BRAF V600E mutation as well as an activating NRAS mutation, but with disparate allele frequencies. Given her age (greater than 60 years old) and 
that activating mutations in NRAS have been identified as arising in the context of clonal hematopoiesis (31), we suspect that her NRAS mutation more likely represents a CHIP-associated somatic mutation. Similarly, the identification of mutations in genes such as DNMT3A and TET2 most likely represent the detection of CHIPassociated genomic alterations, which developed independently of the primary histiocytosis-associated driver mutation. However, further experimental validation is needed to state this conclusively.

Applying molecular diagnostics to the histiocytic disorders may also elucidate the pathogenesis behind disease-associated end-organ damage. For example, in patients with $\mathrm{LCH}$ and bone marrow involvement, CD1a-positive cells are not consistently identified in the bone marrow $(24,32,33)$. In several patients, exemplified by patients 1 and 3 in this report, we detected infiltration of $\mathrm{LCH}$ cells in the bone marrow and liver by the presence of BRAF V600E-positive cells. These cells were CD1a negative, leading us to hypothesize that at least in some cases, LCH may arise from oncogene-mediated transformation of non-Langerhans cells, or that the microenvironment of certain organs (liver, bone marrow, brain) influences the phenotype of the transformed cells, as may be the case in patient 4 . Liver involvement with LCH is generally associated with a poor prognosis, leading to cirrhosis and on occasion the need for liver transplantation. The underlying pathology leading to diffuse liver injury in $\mathrm{LCH}$ has remained a mystery since typical CD1a-positive cells are not consistently identified on biopsies $(6,34)$. The finding of BRAF V600E-positive mononuclear cells in the liver of our patient, however, implicates direct infiltration of transformed LCH cells. Based on the observation that these cells are negative for CD1a and CD163, the cell of origin might be a non-Langerhans monocyte/ macrophage-derived cell, such as a hepatic Kupffer cell, or alternatively, LCH cells may take on a different phenotype in the liver and lose their conventional characteristics such as CD1a positivity. Regardless of the causative cell, the detection of the mutant BRAF protein in addition to laboratory evidence of ongoing liver injury provides further justification for targeted therapy in liver involvement, and provides evidence that liver injury in LCH is caused by direct infiltration of neoplastic cells and is not a paraneoplastic phenomenon.

In summary, this study demonstrates that sequencing-based genomic profiling of histiocytic neoplasms is an important tool to augment conventional histologic diagnosis and identify candidates for targeted therapies, especially in the case of refractory disease. We conclude that MEK inhibition is effective against mutant $M A P 2 K 1$ as well as in cases with $B R A F$ mutations that are resistant to BRAF inhibitors. Given that a significant proportion of these patients relapse or recur despite best available therapies, we propose that all patients with histiocytic neoplasms undergo genomic profiling to identify candidate driver mutations. This facilitates the selection of targeted therapies, sparing the toxicity of more aggressive retrieval chemotherapy regimens. Furthermore, increasing evidence indicates that other mutations found in histiocytic neoplasms, such as the $A L K$ rearrangements, converge on the MAP kinase pathway $(11,35)$. We thus suggest a clinical trial of MEK inhibitors for these disorders, for relapsed/refractory disease, or even potentially as upfront therapy. While clearly effective, the duration of therapy remains unknown. Moreover, reports of disease breakthrough after prolonged treatment with BRAF inhibitors have emerged (36), emphasizing the need for structured clinical trials.

\section{Methods}

Comprehensive genomic profiling. Biopsy tissue of histiocytic lesions was collected and submitted for comprehensive genomic profiling as below as part of routine clinical care. Hybrid capture-based comprehensive genomic and transcriptome profiling was performed using a next-generation sequencing platform as previously described $(37,38)$. Specifically, DNA and RNA were extracted from formalin-fixed, paraffinembedded tissue sections at a CLIA-certified laboratory (Foundation Medicine). DNA sequencing was performed for the entire coding regions of 405 genes and select introns of 31 genes involved in rearrangements, in addition to RNA sequencing of 265 genes using an indexed, adaptor-ligated, hybridization-captured library and sequenced as 49-bp paired-end reads on an Illumina HiSeq 2500 instrument. Sequencing data were processed with a proprietary workflow developed by Foundation Medicine. Alterations with a VAF of $50 \%$ were presumed to be germline, and excluded from our analysis.

Plasmids and cloning. Mutations in $M A P 2 K 1$ or $B R A F$ identified in our patients were generated using site-directed mutagenesis. Primers were designed using the Agilent QuikChange Primer Design Tool (Agilent Technologies). Specific primer pairs for the desired mutations are listed in Supplemental Table 3. Mutations were created in the pBABE-puro-HA-MEK1 vector (Addgene catalog 53195) using procedures detailed in Supplemental Methods.

Cell culture. NIH/3T3 cells (gift from Susa Wells, Cincinnati Children's Hospital) were maintained in Dulbecco's Modifed Eagle Medium (Gibco) supplemented with 10\% fetal calf serum (Gibco), 100 U/ml penicil- 
lin, and $100 \mathrm{mg} / \mathrm{ml}$ streptomycin. BaF/3 cells (gift from Jose Cancelas, Cincinnati Children's Hospital) were maintained in RPMI (Gibco) supplemented with $10 \%$ fetal calf serum and $10 \mathrm{ng} / \mathrm{ml}$ recombinant murine IL-3 (Peprotech). Retroviruses and stable cell lines expressing MEK constructs were generated through the processes described in Supplemental Methods.

Cell growth, focus formation, and soft agar assays. Cell growth in liquid culture was determined by seeding $\mathrm{BaF} / 3$ cells (transduced with vector or MEK1) in triplicate in 24-well plates in IL-3-containing or IL-3-depleted media. Cell viability was determined by trypan blue exclusion. For focus formation assays, $\mathrm{NIH} / 3 \mathrm{~T} 3$ cells $\left(0.5 \times 10^{6}\right)$ stably expressing vector or MEK1 constructs were seeded in triplicate in 6-well plates in monolayer. Media were changed weekly. Bright-field photographs were taken after 21 days using an EVOS microscope (Life Technologies) and foci visible by naked eye were counted.

Immunoblotting. Stably transduced NIH/3T3 cells were grown to near confluence, serum starved for 24 hours, and stimulated with murine EGF (Peprotech) at $50 \mathrm{ng} / \mathrm{ml}$ for 5 minutes (acute stimulation) or indicated time points. Cells were subsequently harvested and protein expression analyzed as described in Supplemental Methods.

Cell line drug treatments. NIH/3T3 cells stably expressing wild-type MEK1 and select mutant MEK constructs were plated in 96-well plates, grown to approximately $80 \%$ confluence, and serum-starved overnight. Cells were subsequently treated for 1 hour with vemurafenib, PD-0325901, AZD-6244 (selumetinib), or trametinib (Selleck Chemicals) in DMSO. For cells transduced with wild-type MEK, drug was removed and cells were stimulated with murine EGF (Peprotech) at $20 \mathrm{ng} / \mathrm{ml}$ for 5 minutes. In-cell Western blotting and imaging (LiCor Biosciences) were performed as detailed in Supplemental Methods. BaF/3 cells stably expressing wild-type or mutant MEK1 were incubated with indicated concentrations of inhibitors targeting BRAF V600E (vemurafenib) or MEK (PD0325901 or trametinib) for 30 minutes in IL-3-containing media. Cells were harvested and probed as described in Supplemental Methods.

Statistics. Statistics were performed with GraphPad Prism 7 software. Significance for Figure 5E was determined by 1 -way ANOVA with Tukey's test post-hoc. $P$ values less than 0.05 were considered significant.

Study approval. This study on human subjects was performed under a protocol reviewed and approved by the Cincinnati Children's Hospital Medical Center IRB under IRB protocol 2016-8780. Requirement for informed consent was waived, as this study involves no more than minimal risk to the human subjects and all data collected arose from procedures and studies which were conducted as part of routine clinical practice.

\section{Author contributions}

Lynn H. Lee, Nicolas N. Nassar, and Ashish R. Kumar conceived and designed the study. Francis X. McCormack, Joseph Pressey, Michael S. Grimley, and Robert Lorsbach provided patient data. Lynn H. Lee, Anjelika Gasilina, Jayeeta Roychoudhury, and Jason Clark performed experiments and assembled experimental data. Siraj Ali, Mark Bailey, Philip Stephens, Jeffrey S. Ross, and Vincent A. Miller provided sequencing and bioinformatics support. All authors were involved in writing the manuscript.

\section{Acknowledgments}

This study was supported in part by funding from the NIH National Heart, Lung, and Blood Institute (R01 HL111192) (to A.R.K.) and the Leukemia and Lymphoma Society grant TRP-6076-14 (to N.N.N.). The authors would like to acknowledge the patients and families who contributed samples to our research efforts. These studies were carried out with support from the DNA sequencing core and the histopathology core at Cincinnati Children's Hospital Medical Center.

Address correspondence to: Ashish Kumar, 3333 Burnet Avenue, Cincinnati, Ohio 45236, USA. Phone: 513.803.2994; E-mail: ashish.kumar@cchmc.org.

1. Delprat C, Aricò M. Blood spotlight on Langerhans cell histiocytosis. Blood. 2014;124(6):867-872.

2. Diamond EL, et al. Consensus guidelines for the diagnosis and clinical management of Erdheim-Chester disease. Blood. 2014;124(4):483-492.

3. Grois N, et al. Central nervous system disease in Langerhans cell histiocytosis. J Pediatr. 2010;156(6):873-81, 881.e1.

4. Gadner H, et al. Therapy prolongation improves outcome in multisystem Langerhans cell histiocytosis. Blood. 2013;121(25):5006-5014. 
5. Donadieu J, et al. Endocrine involvement in pediatric-onset Langerhans' cell histiocytosis: a population-based study. J Pediatr. 2004;144(3):344-350.

6. Braier J, Ciocca M, Latella A, de Davila MG, Drajer M, Imventarza O. Cholestasis, sclerosing cholangitis, and liver transplantation in Langerhans cell histiocytosis. Med Pediatr Oncol. 2002;38(3):178-182.

7. Badalian-Very G, et al. Recurrent BRAF mutations in Langerhans cell histiocytosis. Blood. 2010;116(11):1919-1923.

8. Haroche J, et al. High prevalence of BRAF V600E mutations in Erdheim-Chester disease but not in other non-Langerhans cell histiocytoses. Blood. 2012;120(13):2700-2703.

9. Brown NA, et al. High prevalence of somatic MAP2K1 mutations in BRAF V600E-negative Langerhans cell histiocytosis. Blood. 2014;124(10):1655-1658.

10. Chakraborty R, et al. Mutually exclusive recurrent somatic mutations in MAP2K1 and BRAF support a central role for ERK activation in LCH pathogenesis. Blood. 2014;124(19):3007-3015.

11. Diamond EL, et al. Diverse and targetable kinase alterations drive histiocytic neoplasms. Cancer Discov. 2016;6(2):154-165.

12. Hyman DM, et al. Vemurafenib in multiple nonmelanoma cancers with BRAF V600 mutations. N Engl J Med. 2015;373(8):726-736.

13. Héritier S, et al. Vemurafenib use in an infant for high-risk Langerhans cell histiocytosis. JAMA Oncol. 2015;1(6):836-838.

14. Tzoulis C, et al. Excellent response of intramedullary Erdheim-Chester disease to vemurafenib: a case report. BMC Res Notes. $2015 ; 8: 171$.

15. Brown RA, et al. ETV3-NCOA2 in indeterminate cell histiocytosis: clonal translocation supports sui generis. Blood. 2015;126(20):2344-2345.

16. Bösmüller H, et al. Detection of the BRAF V600E mutation in serous ovarian tumors: a comparative analysis of immunohistochemistry with a mutation-specific monoclonal antibody and allele-specific PCR. Hum Pathol. 2013;44(3):329-335.

17. Capper D, et al. Assessment of BRAF V600E mutation status by immunohistochemistry with a mutation-specific monoclonal antibody. Acta Neuropathol. 2011;122(1):11-19.

18. Long GV, et al. Immunohistochemistry is highly sensitive and specific for the detection of V600E BRAF mutation in melanoma. Am J Surg Pathol. 2013;37(1):61-65.

19. Estep AL, Palmer C, McCormick F, Rauen KA. Mutation analysis of BRAF, MEK1 and MEK2 in 15 ovarian cancer cell lines: implications for therapy. PLoS One. 2007;2(12):e1279.

20. Jeck WR, et al. Targeted next generation sequencing identifies clinically actionable mutations in patients with melanoma Pigment Cell Melanoma Res. 2014;27(4):653-663.

21. Chen SH, et al. Oncogenic BRAF deletions that function as homodimers and are sensitive to inhibition by RAF dimer inhibitor LY3009120. Cancer Discov. 2016;6(3):300-315.

22. Cowley S, Paterson H, Kemp P, Marshall CJ. Activation of MAP kinase kinase is necessary and sufficient for PC12 differentiation and for transformation of NIH 3T3 cells. Cell. 1994;77(6):841-852.

23. Mansour SJ, et al. Transformation of mammalian cells by constitutively active MAP kinase kinase. Science. 1994;265(5174):966-970

24. Berres ML, et al. BRAF-V600E expression in precursor versus differentiated dendritic cells defines clinically distinct LCH risk groups. J Exp Med. 2014;211(4):669-683.

25. Foster SA, et al. Activation mechanism of oncogenic deletion mutations in BRAF, EGFR, and HER2. Cancer Cell. 2016;29(4):477-493.

26. Ohren JF, et al. Structures of human MAP kinase kinase 1 (MEK1) and MEK2 describe novel noncompetitive kinase inhibition. Nat Struct Mol Biol. 2004;11(12):1192-1197.

27. Wagle N, et al. MAP kinase pathway alterations in BRAF-mutant melanoma patients with acquired resistance to combined RAF/MEK inhibition. Cancer Discov. 2014;4(1):61-68.

28. Nelson DS, et al. MAP2K1 and MAP3K1 mutations in Langerhans cell histiocytosis. Genes Chromosomes Cancer. 2015;54(6):361-368.

29. Genovese G, et al. Clonal hematopoiesis and blood-cancer risk inferred from blood DNA sequence. $N$ Engl J Med. 2014;371(26):2477-2487.

30. Xie M, et al. Age-related mutations associated with clonal hematopoietic expansion and malignancies. Nat Med. 2014;20(12):1472-1478.

31. McKerrell T, et al. Leukemia-associated somatic mutations drive distinct patterns of age-related clonal hemopoiesis. Cell Rep. 2015;10(8):1239-1245.

32. Galluzzo ML, Braier J, Rosenzweig SD, Garcia de Dávila MT, Rosso D. Bone marrow findings at diagnosis in patients with multisystem langerhans cell histiocytosis. Pediatr Dev Pathol. 2010;13(2):101-106.

33. Minkov M, Pötschger U, Grois N, Gadner H, Dworzak MN. Bone marrow assessment in Langerhans cell histiocytosis. Pediatr Blood Cancer. 2007;49(5):694-698.

34. Favara BE. Histopathology of the liver in histiocytosis syndromes. Pediatr Pathol Lab Med. 1996;16(3):413-433.

35. Hrustanovic G, et al. RAS-MAPK dependence underlies a rational polytherapy strategy in EML4-ALK-positive lung cancer. Nat Med. 2015;21(9):1038-1047.

36. Gandolfi L, Adamo S, Pileri A, Broccoli A, Argnani L, Zinzani PL. Multisystemic and multiresistant Langerhans cell histiocytosis: a case treated with BRAF inhibitor. J Natl Compr Canc Netw. 2015;13(6):715-718

37. Frampton GM, et al. Development and validation of a clinical cancer genomic profiling test based on massively parallel DNA sequencing. Nat Biotechnol. 2013;31(11):1023-1031.

38. He J, et al. Integrated genomic DNA/RNA profiling of hematologic malignancies in the clinical setting. Blood. 2016;127(24):3004-3014.

39. Cerami E, et al. The cBio cancer genomics portal: an open platform for exploring multidimensional cancer genomics data. Cancer Discov. 2012;2(5):401-404.

40. Gao J, et al. Integrative analysis of complex cancer genomics and clinical profiles using the cBioPortal. Sci Signal. 2013;6(269):pl1. 